\title{
Inspection of magnetization vector and magnetic field distribution of uniaxial and multipole sensor magnets using fast high resolution MagCam magnetic field mapping and analysis
}

\author{
Vervaeke, Koen \\ MagCam NV \\ Research Park Haasrode, Romeinse straat 18, B-3001 Leuven, Belgium
}

\section{Introduction}

Fast and accurate quality inspection of sensor magnets is increasingly important due to higher sensor quality demands and tighter sensor specifications from not only automotive industry [1], but also e.g. consumer electronics industry, where magnetic sensors are increasingly used [2]. Furthermore, in many high-end applications a 100\% magnet quality control is desired, but not feasible. Also in sensor R\&D there is a need for fast and advanced magnet characterization tools for developing next-generation sensor concepts and assemblies $[3,4]$.

Different magnet types require different parameters to be measured and compared to supplier spec sheets for quality control. For uniaxial magnets, the magnetization vector and its deviation angle are important, while for multipole magnets the pole uniformity, symmetry and angular defects are the quality-defining parameters $[5,6]$. Also field homogeneity is important in many applications, such as motor magnets and voice coil motor magnets for hard disk drives [7].

Until now, a fast and high resolution measurement tool for the above characteristics was lacking. Existing magnetic measurement techniques give valuable information, but have limited capabilities, either because they are not suited for small magnets, have limited speed or are not suitable for automation.

We report on a new magnetic measurement technology, called Magnetic Field Camera or "MagCam" [8], a powerful measurement platform for live inspection of different types of permanent magnets. This new concept, using technology knowledge of and developed in the labs of semiconductor company IMEC [9], is optimized for quality control of small magnets, both with uniaxial and multipole magnetizations.

This unique measurement instrument offers unprecedented characterization capabilities for various magnet and magnetic assembly properties. For uniaxial magnets the system measures the $x, y, z$-components of the magnetization vector and directly gives the deviation angle with respect to the geometrical symmetry axis. For multipole magnets an analysis is performed including pole height uniformity, high resolution zero-crossing distance/angle measurements, magnetic asymmetries and more. For magnets where field homogeneity is important, a homogeneity analysis is performed, and field inhomogeneities, due to magnetic or mechanical defects are quantitatively measured with an absolute position indication on the magnet.

\section{Measurement setup}

The measurement setup consists of two main parts, namely a MagCam magnetic field camera sensor module and the measurement \& analysis software, called 'MagScope', as shown in Figure 1. The measurement sample (usually a magnet) is placed on the sensitive sensor surface of the magnetic field camera. The magnetic field camera sensor measures high resolution 'images' of the magnetic field distribution of the magnet at high speed. The MagCam sensor is connected to a computer via a USB-cable, where the MagScope software runs measures and analyzes the magnetic field maps in real time.

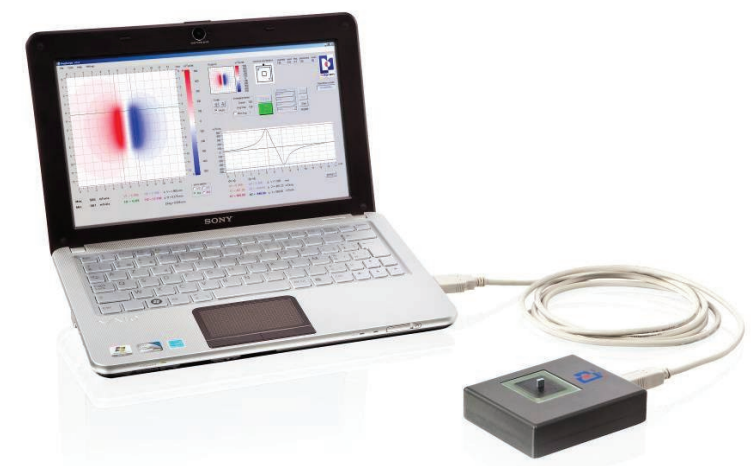

Figure 1: Measurement setup with MagCam magnetic field camera sensor module and MagScope software 


\section{MagCam magnetic field camera sensor hardware}

The MagCam measurement principle is based on a high resolution and high speed quantitative 2D mapping of the magnetic field distribution of the magnet, using a patented sensor chip with an integrated 2D array of $128 \times 128$ (= 16384) microscopic Hall sensors. The sensors have a pitch (resolution) of $0.1 \mathrm{~mm}$ in both $X$ and $Y$ directions. Each Hall sensor has an active area of 40x40 micrometer and locally measures the magnetic field (the perpendicular component). All sensors are electronically scanned at high speed, resulting in a quantitative high resolution magnetic field map over an area of $13 \mathrm{~mm} \times 13 \mathrm{~mm}$. A full resolution MagCam magnetic field map is captured in less than 1 second.
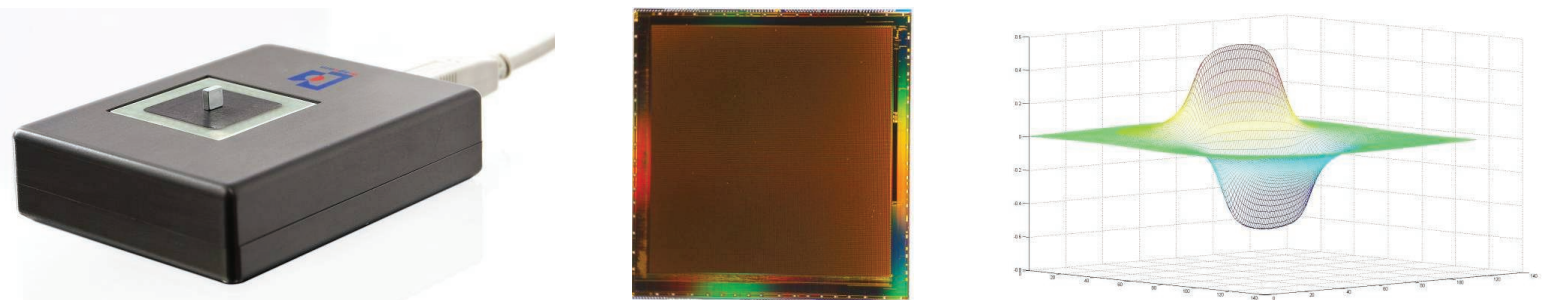

Figure 2: Left: small block magnet $(5 \times 5 \times 2 \mathrm{~mm})$ on the sensitive surface of the MagCam sensor module. The block is uniaxially magnetized along the $2 \mathrm{~mm}$ direction.

Middle: MagCam sensor chip with an integrated 2D array of $128 \times 128$ microscopic Hall sensors.

Right: Surface plot of the measured magnetic field map (Bz component) of the magnet on the left.

The system also allows for absolute magnet positioning, such that the physical magnet position is accurately known relative to the MagCam sensor chip and hence to the measured magnetic field map. This is done using the Magnet Positioning Kit, a set of calibrated frames that can be easily mounted on the MagCam Sensor Module. When a magnet is placed against the frame, its exact position is known in the MagScope software (see e.g. Figure 5).

\section{MagScope and MagFit measurement and analysis software}

The MagScope measurement and analysis software records and analyzes in real time the magnetic field maps measured from the MagCam sensor, as shown in Figure 3. The measured MagCam maps are represented as a high resolution interpolated quantitative color scale graph. This graph can be analyzed using both Cartesian and cylindrical coordinates, depending on the magnet geometry. In Cartesian coordinates, cross-section analyses can be performed across horizontal and vertical lines. In cylindrical coordinates, cross-section analyses can be performed across radial and circumferential lines. Along the cross-section lines, very accurate distance and angle measurements can be made between points of interest in the magnetic field map (e.g. magnetic field zero-crossings, poles, localization of specific magnetic field values, anomalies etc.). These analyses can be performed with absolute magnet position indication. The software can perform image processing algorithms and statistical image analysis in real time.

These features allow to directly characterize a number of magnet properties such as: multipole magnet angle failure, north-south pole asymmetry, magnet (in)homogeneity, material/magnetization defects.

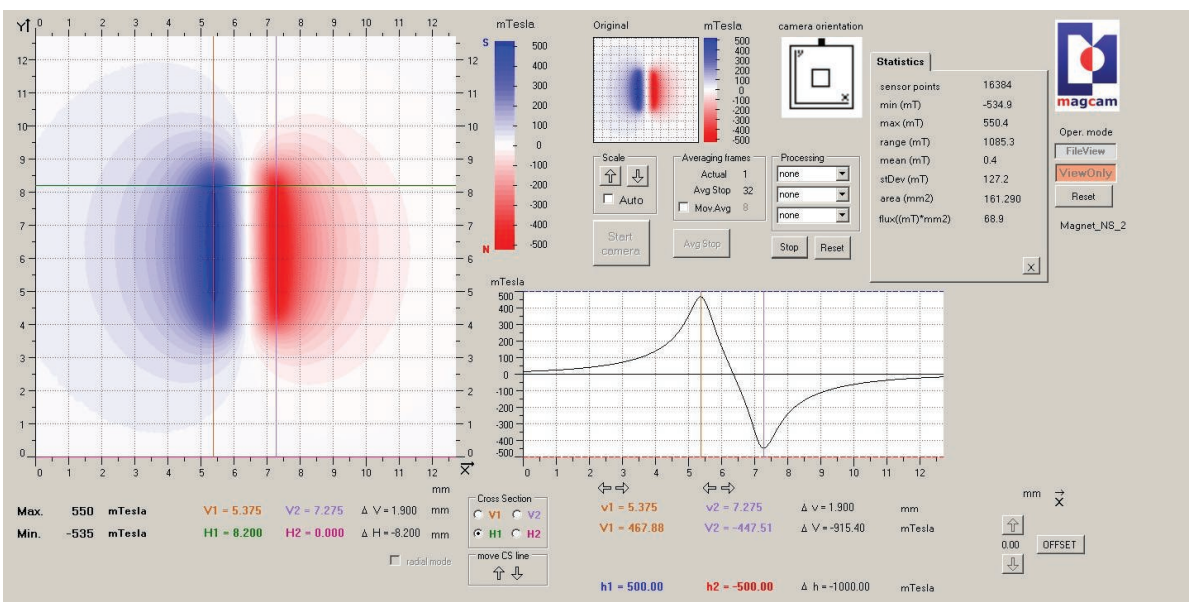

Figure 3: Screenshot of the MagScope measurement and analysis software with left the quantitative interpolated color scale graph $(0.025 \mathrm{~mm}$ pixel resolution) and right the cross-section graph along the green horizontal line on the left. The two vertical lines are positioned precisely onto the magnetic north and south poles and allow measuring their relative distance with $0.025 \mathrm{~mm}$ accuracy. In the upper right area the image statistics are displayed. The complete analysis is performed in real time during measurement or can be performed after recording a MagCam map.

Even more information can be extracted from the MagCam maps using the additional MagFit Magnet Analysis Software module, which offers advanced MagCam data analysis capabilities for a complete characterization of uniaxial permanent magnets. Based on theoretical magnet models, MagFit calculates a best fit to the measured 
MagCam map and can thus extract a lot of extra information from the data, such as the full magnetization vector and its angle deviation, deviations from a 'perfect' magnet, the full B-vector field in 3D space, local defects in the magnetization or in the magnet material, the exact magnet position and orientation, all in a matter of seconds (see Figure 4). All measured parameters can be used for a pass/fail quality control based on user-defined tolerances. Other output includes the fitted (theoretical) MagCam map as well as the residual map (measurement - fit). Examples will be provided further on.

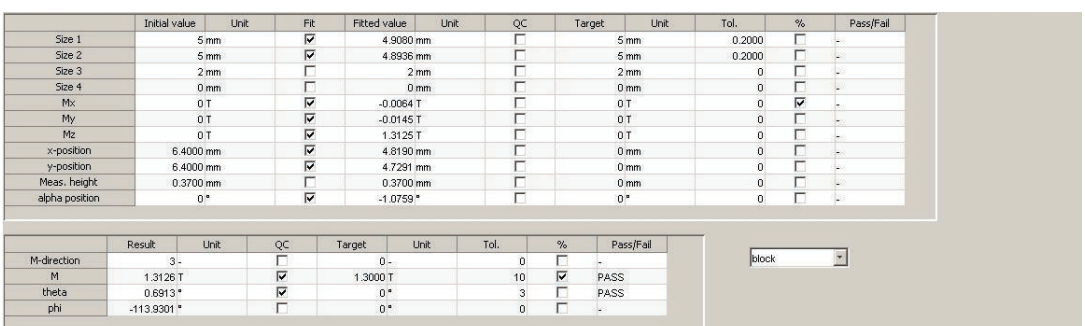

Figure 4: Screenshot of the MagFit magnet analysis software module, showing analysis results for a block magnet such as in Figure 2. The software optimizes a number of parameters in order to obtain a best fit of a theoretical magnet model to the measured MagCam data. Among the output parameters are the full magnetization vector and its deviation angle from the magnet's main geometrical axis, the exact magnet position and orientation, and the magnet sizes. Other output includes the fitted (theoretical) MagCam map.

For use in industrial environments and production lines, the MagCam measurement and analysis can be automated and integrated using an inter-program communication module.

\section{Sensor magnet characterization results}

Using the measurement setup described above, a number of measurements and analyses were performed on different types of commonly used sensor magnets, including both multipole magnets and uniaxially magnetized magnets. A selection of results is provided below.

\section{Multipole sensor magnet inspection}

Small permanent magnets with multipole magnetization patterns are widely used in sensor applications, e.g. for angle or distance measurements. Figure 5 shows an example of a 2-pole cylindrical magnet with axial magnetization, typically used in angular sensor applications. Two important parameters define the quality of such a magnet, namely the angle failure, determined by the border between north and south pole; and the homogeneity of the magnetic field along a circumferential line at a certain radius.

The use of the magnet positioning frame allows to know the exact magnet position and hence the geometrical center of the magnet, which is used as the origin of the cylindrical coordinate system. A circumferential cross-section is taken along the R1 (orange) circle in the color graph. This cross-section is represented in the graph at the right in Figure 5. On this cross-section graph, angles can be accurately and quantitatively measured. Using the two radial lines phi1 (green) and phi2 (purple), the absolute angle deviation of the magnet is measured by taking the angular difference between the two zero-crossing points in the graph. For a perfect magnet, these should be exactly $180^{\circ}$ apart. For this particular magnet, the angle deviation is $12^{\circ}$, a quantitative number that can be directly compared to a (user-defined) tolerance for pass/fail quality assessment.

The second quality-defining parameter, namely the magnetic field homogeneity, is readily observed both in the color graph and in the cross-section graph. Threshold values indicating tolerances can be set for detecting failing magnet areas. In Figure 5, the magnetic field shows an inhomogeneity around $30^{\circ}$ (see cross-section graph, where the magnetic field drops below the blue reference line).
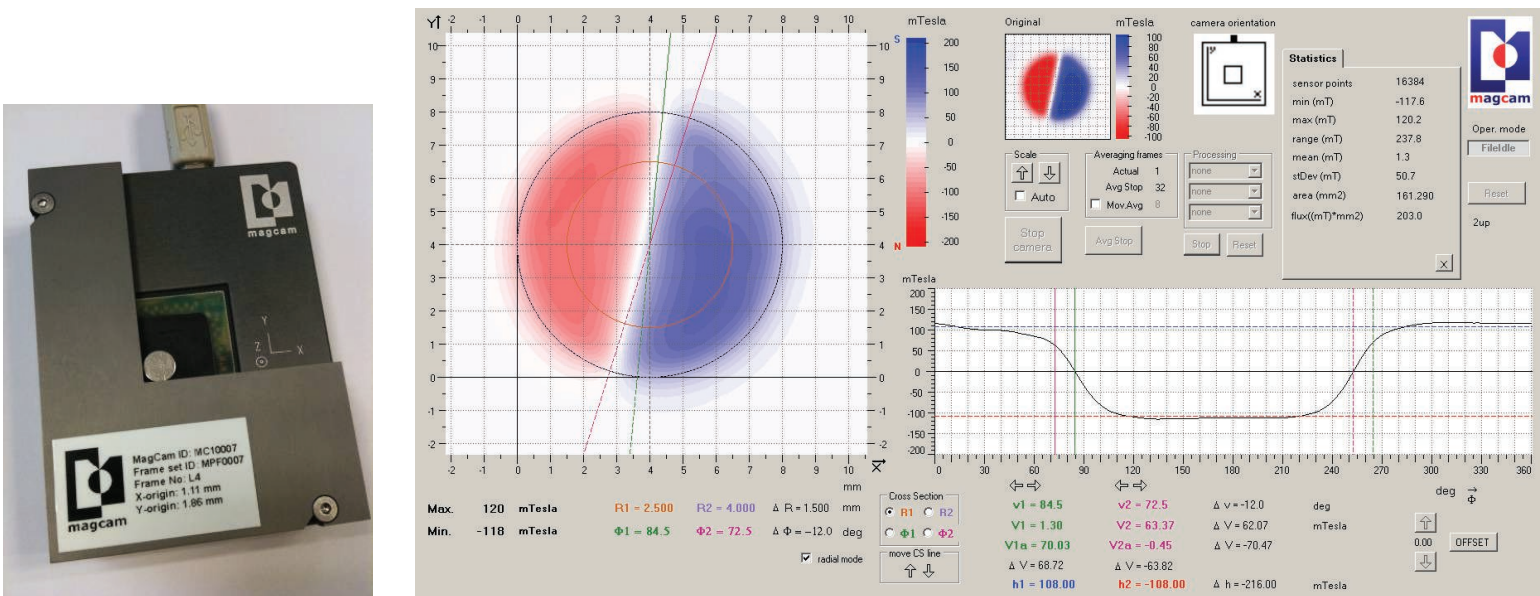

Figure 5: Left: photograph of an 8mm diameter cylindrical multipole magnet on the MagCam sensor using a calibrated magnet positioning frame. Right: MagScope screenshot showing the measurement and analysis result of 
the magnet on the left. Using exact position information and cylindrical coordinate analysis, the angle failure, pole symmetry and homogeneity of the magnet can be readily determined and tested against specified tolerances.

This example shows how multipole magnets can be inspected in a very detailed and economical way using the magnetic field camera principle.

\section{Uniaxial sensor magnet inspection}

Uniaxially magnetized magnets are the most common form of permanent magnets and are also widely used in sensor applications. Typical magnet materials include SmCo, Ferrite, AINiCo and NdFeB. A uniaxial magnet typically has a basic geometrical shape (block, disk, ring, segment) with one magnetization axis which is usually along one of the main geometrical axes or perpendicular to it. Especially NdFeB magnets suffer from a deviation of the magnetization axis relative to the geometrical magnet axis. This is due to the crystalline nature of the material, which crystal axes are not always parallel to the geometrical axes of the machined magnet. This deviation can be characterized by the angle the magnetization vector makes with the geometrical main axis. This 'angle deviation' or 'skew angle' directly translates itself into measurement deviations when the magnet is built into a sensor assembly. The angle deviation is therefore one of the most important characteristics of a uniaxial sensor magnet and should be as small as possible (ideally $0 \%$. For small magnets however, traditional magnetization measurement equipment, such as Helmholtz coils, become inaccurate due to the small amount of material in the magnet. Moreover, Helmholtz coils only measure one parameter, i.e. the magnetization vector.

The measurements and analysis in this section show that the MagCam setup is able to measure the full magnetization vector and its deviation angle. On top of that, also the magnet's size, position and orientation are obtained. This is done using an advanced data analysis algorithm that fits a theoretical magnet model to the measured MagCam data and in the process optimizes a number of model parameters to obtain a best fit theoretical MagCam map. This theoretical MagCam map is also available as output from the analysis.

Moreover, when subtracting the theoretical map from the measured map, a 'residual' map is obtained, which shows all deviations of the measured MagCam map from the theoretical best fit map. On this residual map local deviations from the 'perfect' magnet are very clearly visible. These deviations can be due to material defects such as impurities or structural defects in the magnet material, or defects in the magnetization of the magnet. This residual map is perfectly suited to run a statistics analysis (see further), yielding quantitative characteristics of the deviation of the magnet from a perfect magnet. These image statistics can in turn be used to be tested against user-defined tolerance limits for e.g. a pass/fail quality control.

In the example below a uniaxially magnetized NdFeB block magnet $(5 \times 5 \times 2 \mathrm{~mm})$ is measured using the MagCam setup (Figure 6) and its MagCam map is subsequently analyzed using the MagFit software (Figure 7), yielding a set of optimized parameters which can be regarded as the measured values for this magnet. Among these parameters are the magnetization vector components, its size and its angle deviation, the magnet dimensions, and the magnet position and orientation on the sensor.
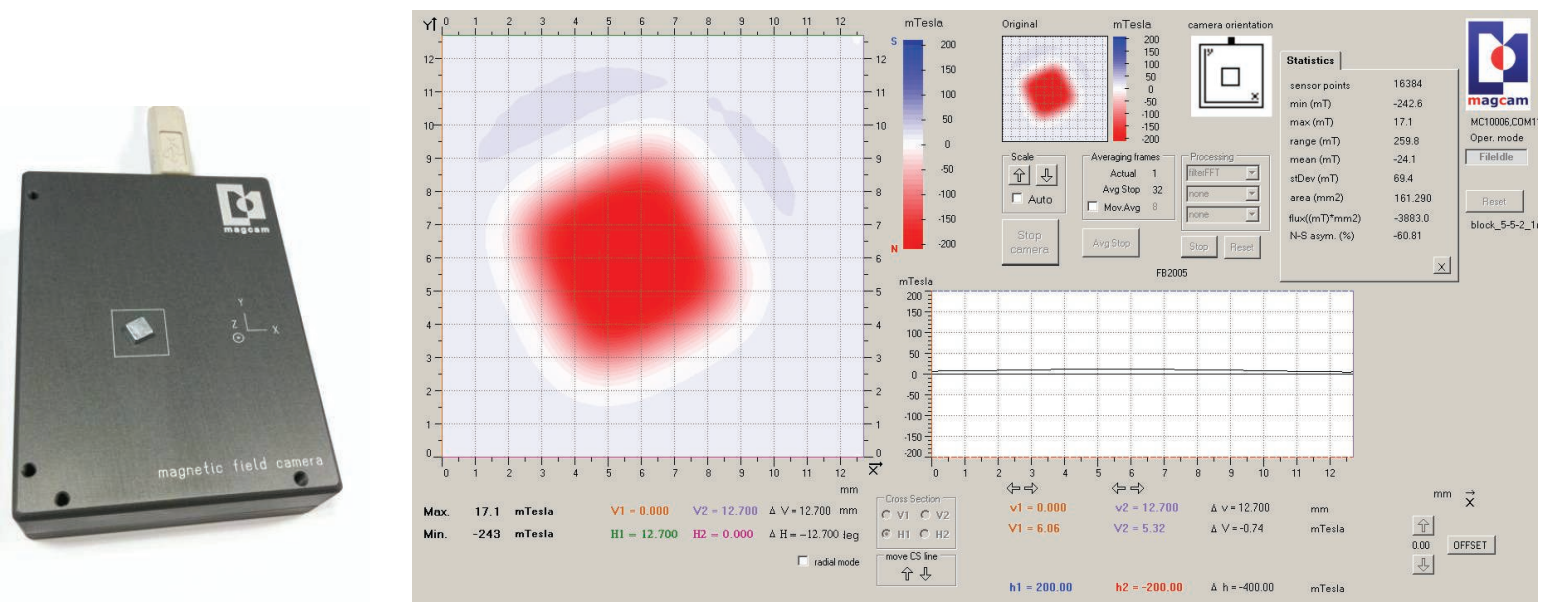

Figure 6: Left: photograph of a 5x5x2mm block magnet (NdFeB) on the MagCam sensor. Right: MagScope screenshot showing the MagCam map of the magnet on the left (the north pole is on the sensor surface).

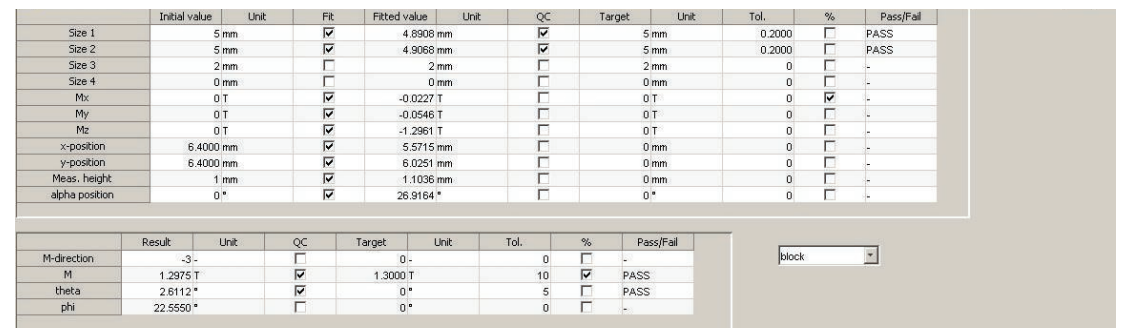


Figure 7: MagFit analysis of the MagCam map from Figure 6, showing the initial values and optimized values ('Fitted value' column) of the fitting parameters in the optimization procedure. Fitting parameters include the lateral sizes of the block magnet (Size1, Size 2), the three Cartesian components of the magnetization vector (Mx,My,Mz), the lateral position of the magnet's center on the sensor ( $x$-position, $y$-position) and the angular position of the magnet on the sensor. In the table below the magnetization vector components are converted to spherical coordinates, automatically yielding the main magnet axis (note that the initial parameters for $M x, M y, M z$ are 0,0,0), the absolute value of the magnetization (1.298T in this case) and the deviation of the magnetization vector from the main axis (theta $=2.61 \%$. The fitted parameters can be tested against user-defined tolerance limits for pass/fail quality control.

The next example below shows a MagCam measurement and subsequent MagFit analysis of an axially magnetized NdFeB disk magnet $(D=12 \mathrm{~mm}, h=0.4 \mathrm{~mm})$. No magnet positioning frame is used for the measurement.

Nevertheless, the absolute position of the magnet is known since the position of the magnet's center is one of the resulting output parameters of the MagFit optimization. This magnet center position is subsequently used as the origin of the cylindrical coordinate system in the MagScope analysis in Figure 8 and Figure 10. After the MagFit analysis (Figure 9), the resulting theoretical best fit map and residual map are viewed and analyzed using the MagScope software (Figure 10). This residual map only contains the magnet's 'imperfections' and shows an amplified image of the material defect (crack) that is present in the magnet. On this residual image, image statistics (e.g. magnetic field range, standard deviation etc.) give relevant information and can be used for automatic and objective pass/fail quality control.

Note that in the fitted (theoretical) MagCam map the angle deviation of the magnetization vector is already taken into account, so it is not present in the residual map. If one wishes, one could also exclude the angle deviation from the MagFit analysis (by unchecking the 'fit' option for Mx and My in Figure 9). The angle deviation would then become apparent in the residual map as an asymmetry in the image in the 'phi' direction shown in the lower table in Figure 9. Moreover, once the input parameters for the magnet model have been optimized, one has a full theoretical magnet model that can be used to calculate all magnetic field components $(\mathrm{Bx}, \mathrm{By}, \mathrm{Bz})$ at any point in space, even though the MagCam measures only the Bz component in one plane in space!
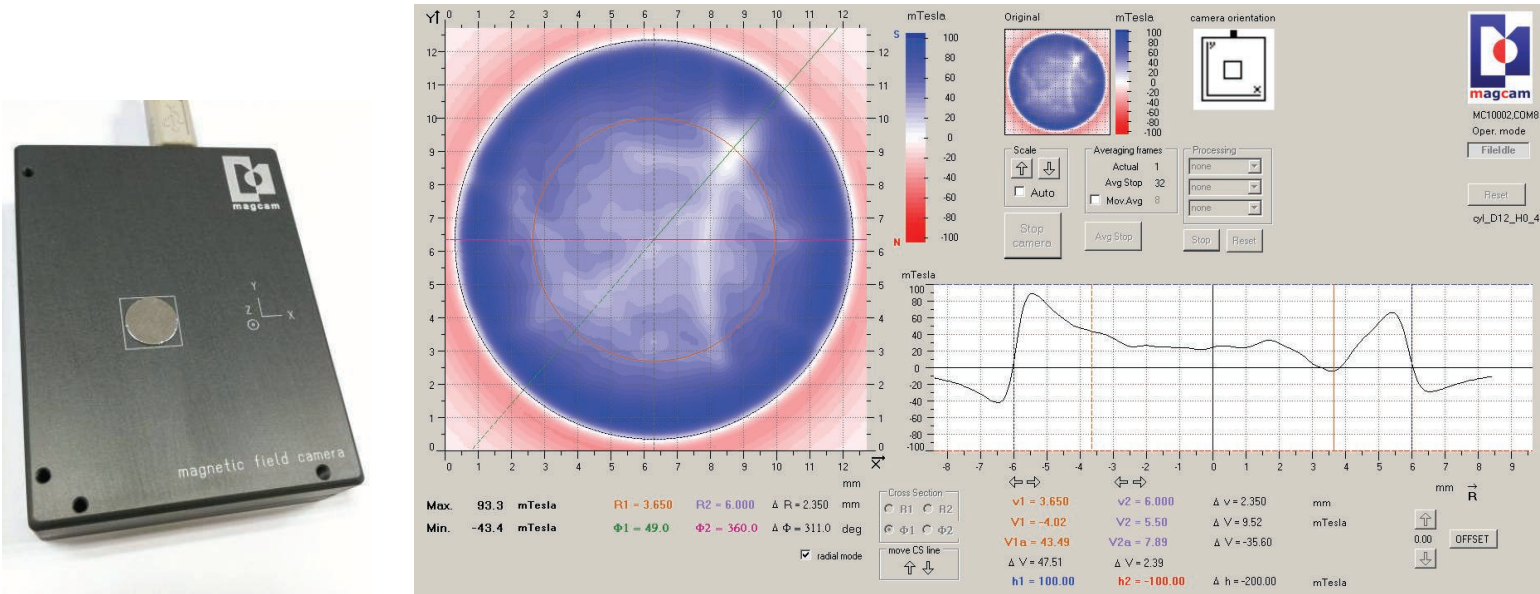

Figure 8: Left: photograph of a 12mm diameter, 0.4mm high disk magnet (NdFeB) on the MagCam sensor. Right: MagScope screenshot showing the MagCam map of the magnet on the left (the south pole is on the sensor surface). A clear inhomogeneity is observed in the magnetic field distribution, caused by a physical crack inside the magnet. The cross-section graph displays the cross-section along the green radial line (phi1) going through the defect at an angular position of $49.0^{\circ}$. This inhomogeneity is amplified in the residual map after MagFit analysis (see Figure 10).

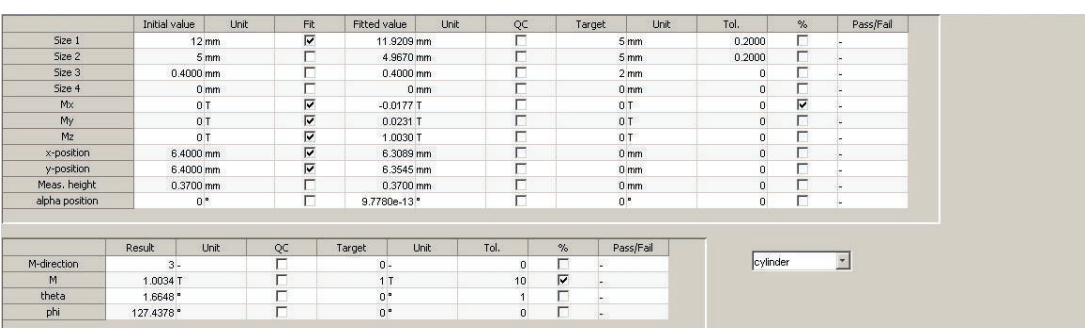

Figure 9: MagFit analysis of the MagCam map from Figure 8, showing the initial values and optimized values ('Fitted value' column) of the fitting parameters in the optimization procedure. Fitting parameters include the diameter of the block magnet (Size1), the three Cartesian components of the magnetization vector $(M x, M y, M z)$ and the lateral position of the magnet's center on the sensor (x-position, $y$-position). The angular position is not relevant for an axially magnetized disc due to symmetry. In the table below the magnetization vector components are converted to spherical coordinates, automatically yielding the main magnet axis (note that the initial parameters for $M x, M y, M z$ are $0,0,0)$, the absolute value of the magnetization (1.003T in this case) and the deviation of the magnetization vector from the main axis (theta $=1.66 \%$. The fitted parameters can be tested against user-defined tolerance limits for pass/fail quality control. 

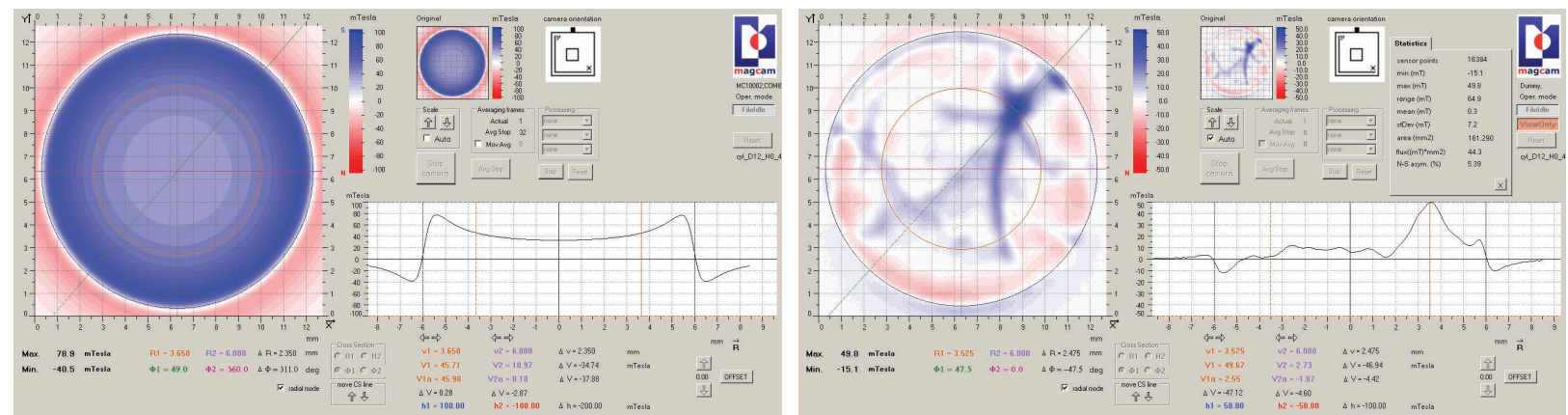

Figure 10: Left: Theoretical best fit MagCam map resulting from the MagFit analysis. This map is completely noisefree (theoretical calculation) and free of 'defects', except for the magnetization vector angle deviation, which is included in the map since the $M x$ and My components of the magnetization vector were included in the optimization procedure.

Right: Residual MagCam map (theoretical map - measured map). In this image, the background of the 'perfect' theoretical magnet on the left is removed from the measured MagCam map, leaving only all 'imperfections' in the magnetic field distribution. These 'imperfections' can indicate inhomogeneities and material/magnetization defects. In this particular magnet, a large inhomogeneity is present, due to a crack in the magnet material. The residual MagCam map is very well suited for analysis using image statistics, yielding objective and quantitative results that can be tested against user-defined tolerance limits for e.g. pass/fail quality control.

\section{Conclusion}

Driven by anindustrial need for advanced inspection equipment for (small) permanent magnets, such as the highquality magnets used in sensor applications, we have presented a new magnetic measurement technology, called the Magnetic Field Camera or 'MagCam', a powerful and unique measurement platform for fast and accurate live inspection of both uniaxial and multipole permanent magnets. Measurements and analyses on both multipole and uniaxial magnets were performed, showing the capabilities of the system to perform a full characterization of small permanent magnets, including the quality-defining characteristics such as angle failure, pole symmetry and homogeneity for multipole magnets and magnetization vector size and angle deviation for uniaxial magnets. Using advanced data analysis and combining theoretical magnet modeling and MagCam measurements, a lot of extra information can be extracted from the measurement data, including the magnet's exact position and orientation, the magnetization vector, a theoretical best fit to the MagCam map and a residual MagCam map showing all 'imperfections' in the magnet.

This powerful magnet inspection platform is well suited for both R\&D and industrial (automated) magnet inspection, since it is completely digital gives fast measurement and analysis results (in seconds), and can be remotely controlled. It is therefore the author's belief that the presented measurement system can become a new industry standard for (sensor) magnet inspection.

\section{References}

[1] www.melexis.com , Magnet Selection Application Note MLX90316

[2] V Sankaranarayanan, Magnetic Sensors Expand their Reach from Automotive to Cell Phones and Consumer Electronics Applications, Frost \& Sullivan Market Research Report (2010)

[3] U. Ausserlechner, Inaccuracies of Giant Magneto-Resistive Angle Sensors Due to Assembly Tolerances, IEEE Trans. Magn. 45, No 5, 2165 (2009)

[4] www.arnoldmagnetics.com , Technical papers and presentations, Polymer Bonded Magnets

[5] Y.N. Zhilichev, Precise Multipole Magnetization of Disk Magnet for Sensor Applications, IEEE Trans. Magn. 39, No 5, 3301 (2003)

[6] J. Paulides et al., Sinusoidal Behavior of a Dipole Magnetization for Position Sensing Applications, IEEE Trans. Magn., Vol. 42, No. 10, 3294 (2006)

[7] G. Ombach, Electrical Motors for Automotive Applications, Trends and Requirements on Magnetic Materials, Proceedings of WMM'10 Conference, Freiberg, Germany (2010)

[8] www.magcam.com , Technology page

[9] K. Vervaeke, Microscopic Hall sensors for Magnetometry and Local Magnetic Imaging, Ph.D. Thesis at the University of Leuven, Belgium (2007) 